\title{
User-friendly Rshiny web applications for supporting syndromic surveillance analysis
}

\author{
Anne Fouillet*, Marc Ruello, Lucie Léon, Cécile Sommen, Laurent Marie, \\ Céline Caserio-Schönemann, Camille Pelat and Yann Le Strat
}

Santé publique France, Saint-Maurice, France

\section{Objective}

The presentation describes the design and the main functionalities of two user-friendly applications developed using R-shiny to support the statistical analysis of morbidity and mortality data from the French syndromic surveillance system SurSaUD.

\section{Introduction}

The French syndromic surveillance system SursaUD ${ }^{\circledR}$ has been set up by Santé publique France, the national public health agency (formerly French institute for public health - InVS) in 2004. In 2016, the system is based on three main data sources: the attendances in about 650 emergency departments (ED), the consultations to 62 emergency general practitioners' (GPs) associations SOS Médecins and the mortality data from 3,000 civil status offices [1].

Daily, about 60,000 attendances in ED ( $88 \%$ of the national attendances), 8,000 visits in SOS Médecins associations (95\% of the national visits) and 1,200 deaths ( $80 \%$ of the national mortality) are recorded all over the territory and transmitted to Santé publique France.

About 100 syndromic groupings of interest are constructed from the reported diagnostic codes, and monitored daily or weekly, for different age groups and geographical scales, to characterize trends, detect expected or unexpected events (outbreaks) and assess potential impact of both environmental and infectious events. All-causes mortality is also monitored in similar objectives.

Two user-friendly interactive web applications have been developed using the $\mathrm{R}$ shiny package [2] to provide a homogeneous framework for all the epidemiologists involved in the syndromic surveillance at the national and the regional levels.

\section{Methods}

The first application, named MASS-SurSaUD, is dedicated to the analysis of the two morbidity data sources in Sursaud, along with data provided by a network of Sentinel GPs [3]. Based on pre-aggregated data availaible daily at 10:30 am, R programs create daily, weekly and monthly time series of the proportion of each syndromic grouping among all visits/attendances with a valid code at the national and regional levels. Twelve syndromic groupings (mainly infectious and respiratory groups, like ILI, gastroenteritis, bronchiolitis, pulmonary diseases) and 13 age groups have been chosen for this application.

For ILI, 3 statistical methods (periodic regression, robust periodic regression and Hidden Markov model) have been implemented to identify outbreaks. The results of the 3 methods applied to the 3 data sources are combined with a voting algorithm to compile the influenza alarm level for each region each week: non-epidemic, pre/post epidemic or epidemic.

The second application, named MASS-Euromomo, allows consulting results provided by the model developed by the European project EuroMomo for the common analysis of mortality in the European countries (www.euromomo.eu). The Euromomo model, initially developed using Stata software, has been transcripted in R. The model has been adapted to run in France both at a national, regional and other geographical administrative levels, and for 7 age groups.

\section{Results}

The two applications, accessible on a web-portal, are similarly designed, with:

- a dropdown menu and radio buttons on the left hand side to select the data to display (e.g. filter by data source, age group, geographical levels, syndromic grouping and/or time period),

- several tab panels allowing to consult data and statistical results through tables, static and dynamic charts, statistical alarm matrix, geographical maps,... (Figure 1),

- a "help" tab panel, including documentations and guidelines, links, contact details.

The MASS-SurSaUD application has been deployed in December 2015 and used during the 2015-2016 influenza season. MASSEuromomo application has been deployed in July 2016 for the heatwave surveillance period. Positive feedbacks from several users have been reported.

\section{Conclusions}

Business Intelligence tools are generally focused on data visualisation and are not generally tailored for providing advanced statistical analysis. Web applications built with the R-shiny package combining user-friendly visualisations and advanced statistics can be rapidly built to support timely epidemiological analyses and outbreak detection.

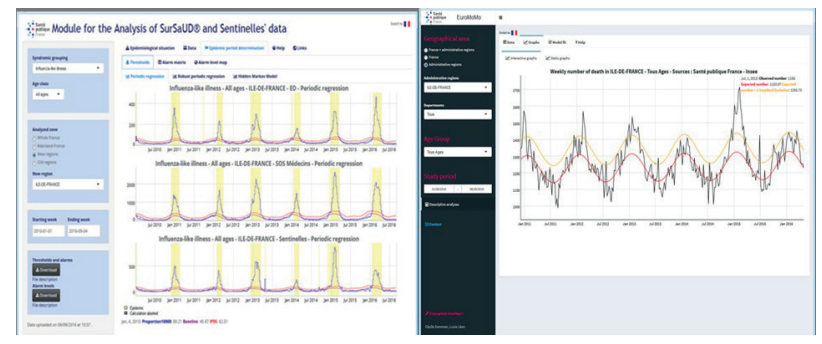

Figure 1: screen-shots of a page of the two applications

\section{Keywords}

shiny; user-friendly application; France; SurSaUD

\section{References}

[1] Caserio-Schönemann C, Bousquet V, Fouillet A, Henry V. The French syndromic surveillance system SurSaUD. Bull Epidémiol Hebd 2014;3-4:38-44.

[2] Chang, W., Cheng, J., Allaire, J., Xie, Y., \& McPherson, J. (2015). Shiny: web application framework for R. R package version 0.11, 1 .

[3] Valleron AJ, Bouvet E, Garnerin P, Ménarès J, Heard I, Letrait S, Lefaucheux J. A computer network for the surveillance of communicable diseases: the French experiment. Am J Public Health. 1986. 76(11):1289-92

\section{*Anne Fouillet \\ E-mail: a.fouillet@invs.sante.fr}

